\title{
MicroRNA evolution, expression, and function during short germband development in Tribolium castaneum
}

\author{
Maria Ninova, Matthew Ronshaugen, and Sam Griffiths-Jones \\ Faculty of Life Sciences, University of Manchester, Manchester, M13 9PT, United Kingdom
}

\begin{abstract}
MicroRNAs are well-established players in the development of multicellular animals. Most of our understanding of microRNA function in arthropod development comes from studies in Drosophila. Despite their advantages as model systems, the long germband embryogenesis of fruit flies is an evolutionary derived state restricted to several holometabolous insect lineages. MicroRNA evolution and expression across development in animals exhibiting the ancestral and more widespread short germband mode of embryogenesis has not been characterized. We sequenced small RNA libraries of oocytes and successive intervals covering the embryonic development of the short germband model organism, Tribolium castaneum. We analyzed the evolution and temporal expression of the microRNA complement and sequenced libraries of total RNA to investigate the relationships with microRNA target expression. We show microRNA maternal loading and sequence-specific $3^{\prime}$ end nontemplate oligoadenylation of maternally deposited microRNAs that is conserved between Tribolium and Drosophila. We further uncover large clusters encoding multiple paralogs from several Tribolium-specific microRNA families expressed during a narrow interval of time immediately after the activation of zygotic transcription. These novel microRNAs, together with several early expressed conserved microRNAs, target a significant number of maternally deposited transcripts. Comparison with Drosophila shows that microRNA-mediated maternal transcript targeting is a conserved process in insects, but the number and sequences of microRNAs involved have diverged. The expression of fast-evolving and species-specific microRNAs in the early blastoderm of $T$. castaneum is consistent with previous findings in Drosophila and shows that the unique permissiveness for microRNA innovation at this stage is a conserved phenomenon.
\end{abstract}

[Supplemental material is available for this article.]

MicroRNAs are short nonprotein-coding RNAs, processed from hairpin precursors. MicroRNAs regulate gene expression by guiding the RNA-induced silencing complex (RISC) to complementary sites in the $3^{\prime}$ UTRs of target mRNAs, thereby inducing translational silencing and degradation (for review, see Bartel 2004). MicroRNA-target interaction usually requires base-pairing within a 6- to 7-mer "seed" region at the 5' end of the mature microRNA, and thus each microRNA can potentially target hundreds of protein-coding genes (for review, see Bartel 2009). Consistent with their functional importance, seed sequences are the most highly conserved regions of the microRNA hairpins (Lai et al. 2003; Lim et al. 2003a,b). MicroRNAs were first identified for their role in the regulation of developmental timing in Caenorhabditis elegans (Lee et al. 1993; Reinhart et al. 2000) and were later shown to play important roles in various aspects of the development of both invertebrates and vertebrates (for review, see Kloosterman and Plasterk 2006). Most of our understanding of microRNA function in the development of arthropods comes from studies in the classical model organism Drosophila melanogaster, where they control essential developmental processes such as the clearance of maternally deposited transcripts, cell differentiation and apoptosis, morphogenesis, and organogenesis (Asgari 2013).

Corresponding authors: sam.griffiths-jones@manchester.ac.uk, matthew.ronshaugen@manchester.ac.uk

Article published online before print. Article, supplemental material, and publication date are at http://www.genome.org/cgi/doi/10.1101/gr.193367.115. Freely available online through the Genome Research Open Access option.
Despite its advantages as a model species, Drosophila development is not representative of the vast majority of arthropods. Fruit flies follow the so-called "long germband" developmental mode, which is derived and found only in a subset of holometabolous insect lineages (Peel 2008; Mito et al. 2010). The most common, and likely ancestral, mode of arthropod presegmentation development is short germband embryogenesis (Peel 2008; Mito et al. 2010). In short germband embryogenesis, a small number of cells in the blastoderm (called the germ anlage) form the most anterior embryonic segments, and the remaining portion gives rise to the extraembryonic serosal membrane (Handel et al. 2000). Whereas long germ development often occurs in a syncytium, more posterior segments in short-germband embryogenesis arise after gastrulation by growth and cell division, and patterning occurs in a cellularized environment via an oscillatory mechanism (Sarrazin et al. 2012). In many respects, short germband embryogenesis therefore more closely resembles the segmentation of vertebrate embryos.

The red flour beetle Tribolium castaneum is an emerging model organism that displays a number of ancestral features, including the short germband mode of development (Denell 2004; Richards et al. 2008; Roth and Hartenstein 2008). The availability of genetic tools and a wide range of embryonic patterning mutants have established T. castaneum as a model system to study this ancestral developmental mode (Denell 2004; Richards et al. 2008). $T$. castaneum has a fully sequenced genome (Richards et al. 2008)

(C) 2016 Ninova et al. This article, published in Genome Research, is available under a Creative Commons License (Attribution 4.0 International), as described at http://creativecommons.org/licenses/by/4.0/. 
and an annotated protein-coding transcriptome (Kim et al. 2010). In addition, the morphology of its early embryogenesis is among the best characterized of the short germband insects (Handel et al. 2000, 2005; Denell 2004; Benton et al. 2013).

MicroRNAs are recognized as important players in developmental gene regulation, yet their expression and function in short-germband embryogenesis is poorly understood. Furthermore, little is known about the evolutionary constraints that act on microRNA developmental expression in general. We explored the microRNA complement of $T$. castaneum and expression of their targets in a developmental context, using a combination of small RNA and whole transcriptome RNA sequencing of successive time intervals covering beetle embryogenesis. We find that microRNA abundance markedly increases at the onset of zygotic transcription, both for conserved microRNAs and a large number of previously unannotated microRNAs organized in multiple rapidly evolving multicopy clusters. We show that maternally deposited protein-coding mRNAs that are down-regulated in the early embryo are significantly enriched in targets of these up-regulated microRNA families. We therefore show a role for early expressed microRNAs in maternal transcript clearance. Comparison with previous findings of microRNA-mediated maternal transcript degradation in Drosophila (Bushati et al. 2008) allows unprecedented insights into the evolution, expression, and function of microRNAs in the early stages of arthropod development.

\section{Results}

\section{T. castaneum small RNA sequencing and annotation}

T. castaneum embryonic development is significantly longer than that of fruit flies, spanning $6 \mathrm{~d}$ at $25^{\circ} \mathrm{C}$. We collected embryonic samples from different time intervals after egg laying to determine the time points when key developmental events occurred (Supplemental Text; Supplemental Fig. 1). Based on these observations, we generated small RNA sequencing libraries from seven discrete intervals of $T$. castaneum embryogenesis covering the following key stages: very early embryo before the onset of zygotic transcription ( $0-5 \mathrm{~h}$, herein referred to as "pre-ZT" embryos; note that zygotic transcription occurs at $\sim 8 \mathrm{~h}$ ) (Supplemental Fig. 1B); later cleavage divisions and blastoderm formation (8-16 h); blastoderm differentiation and beginning of gastrulation (16-20 h); progressing serosal closure (20-24 h); elongating germband (24-34 h); fully segmented germband and appendage formation onset (34-48 h); and extended germband until hatching (48-144 h). We obtained between 3.5 and 6.5 million reads for each sample, over $85 \%$ of which mapped to the $T$. castaneum genome with no more than one mismatch.

The previously annotated set of microRNAs in T. castaneum comprises 203 hairpins that were experimentally identified in mixed adult and mixed embryonic data sets. Nearly half of these sequences have no identifiable orthologs in other species with sequenced genomes (Supplemental Table 1; Marco et al. 2010). We took advantage of the discrete embryonic stage data sets to search for putative novel microRNAs that may be expressed during narrow periods of time and thus escaped previous detection. We revised previous annotations and uncovered a total of 123 novel Tribolium-specific microRNA candidates using two independent approaches for novel microRNA identification (see Supplemental Table 1). A large fraction of these hairpins are paralogs of, or have similar extended seed sequences to, the microRNA families mir-3851 and mir-3836. Homology searches suggest that the mir-3851 family is Tribolium-specific (Supplemental Fig. 2). The miR-3836-3p has a seed sequence identical to that of the miR-3 family, but there is little similarity outside the seed, so homology cannot be confidently assigned. Regardless of their ancestral origins, the large number of paralogs from the mir-3836 family in T. castaneum suggests that they emerged by multiple lineage-specific duplications. Furthermore, there is a second set of novel sequences that lack homology with any known microRNAs. Many of these sequences are found in multiple copies and can be grouped in several families (Supplemental Fig. 2). Notably, the vast majority of mir-3851, mir-3836, and novel microRNA family members are organized in clusters in the genome, mostly localized in subtelomeric regions or unmapped scaffolds (Fig. 1). These clusters differ in their members' copy number, sequence, and organization, suggesting rapid evolution by duplication and diversification. We collectively refer to these clusters as "multicopy microRNA clusters."

\section{Small RNA temporal dynamics during T. castaneum embryogenesis}

To gain insight into the developmental dynamics of small RNAs during T. castaneum development, we first assessed the overall content of the small RNA sequencing libraries of successive time intervals. Figure 2 shows the distributions of small RNA reads in each embryonic stage. Reads from all libraries and sizes display a very strong bias for uracil in the first position, which is typical for microRNAs and piRNAs. Size distributions of the sequenced reads display two peaks at $\sim 22$ and $\sim 28 \mathrm{nt}$, with the vast majority of the $\sim 22$-nt reads corresponding to known or newly annotated microRNAs. Thorough examination of the $\sim 28$-nt fraction showed that these represent an abundant piRNA fraction (M Ninova, S Griffiths-Jones, M Ronshaugen, in prep.). The relative levels of putative microRNAs and piRNAs change markedly as development progresses. piRNAs are highly abundant in the earliest stages of development, consistent with their maternal deposition (Brennecke et al. 2008). MicroRNA levels, on the other hand, are initially very low but gradually increase and account for nearly $30 \%$ of all small RNA reads in the late embryo. These figures are in sharp contrast with the small RNA distributions observed in early embryonic developmental data sets from Drosophila, where microRNAs represent a significant fraction of the early embryonic small RNAs (see below) and dominate past the first couple of hours of development.

\section{Abundance, diversity, conservation, and $3^{\prime}$ end modification of maternally deposited microRNAs}

We hypothesized that the apparent low microRNA levels in T. castaneum pre-ZT embryos reflects either insignificant maternal loading of microRNAs in oocytes or decreased sampling of microRNAs by sequencing due to high piRNA levels. We therefore sought to determine the absolute amount of microRNAs deposited in Tribolium and Drosophila eggs. To this end, we sequenced samples prepared from a fixed number of unfertilized eggs from $T$. castaneum and two divergent fruit fly species, D. melanogaster and $D$. virilis, with spiked-in synthetic 5 '-phosphorylated oligonucleotides for normalization (see Supplemental Text; Methods). In addition, we quantified the absolute cellular levels of the abundant miR-184-3p and used it as an additional endogenous reference. miR-184-3p and spike-in levels were in a good agreement between qPCR and sequencing estimates, confirming that the sequencing data reflect well the microRNA abundances in this concentration range (Supplemental Text; Supplemental Fig. 3). Small RNA size distributions of the resulting libraries are shown in Figure 3A. As expected, T. castaneum oocytes show similar profiles to 

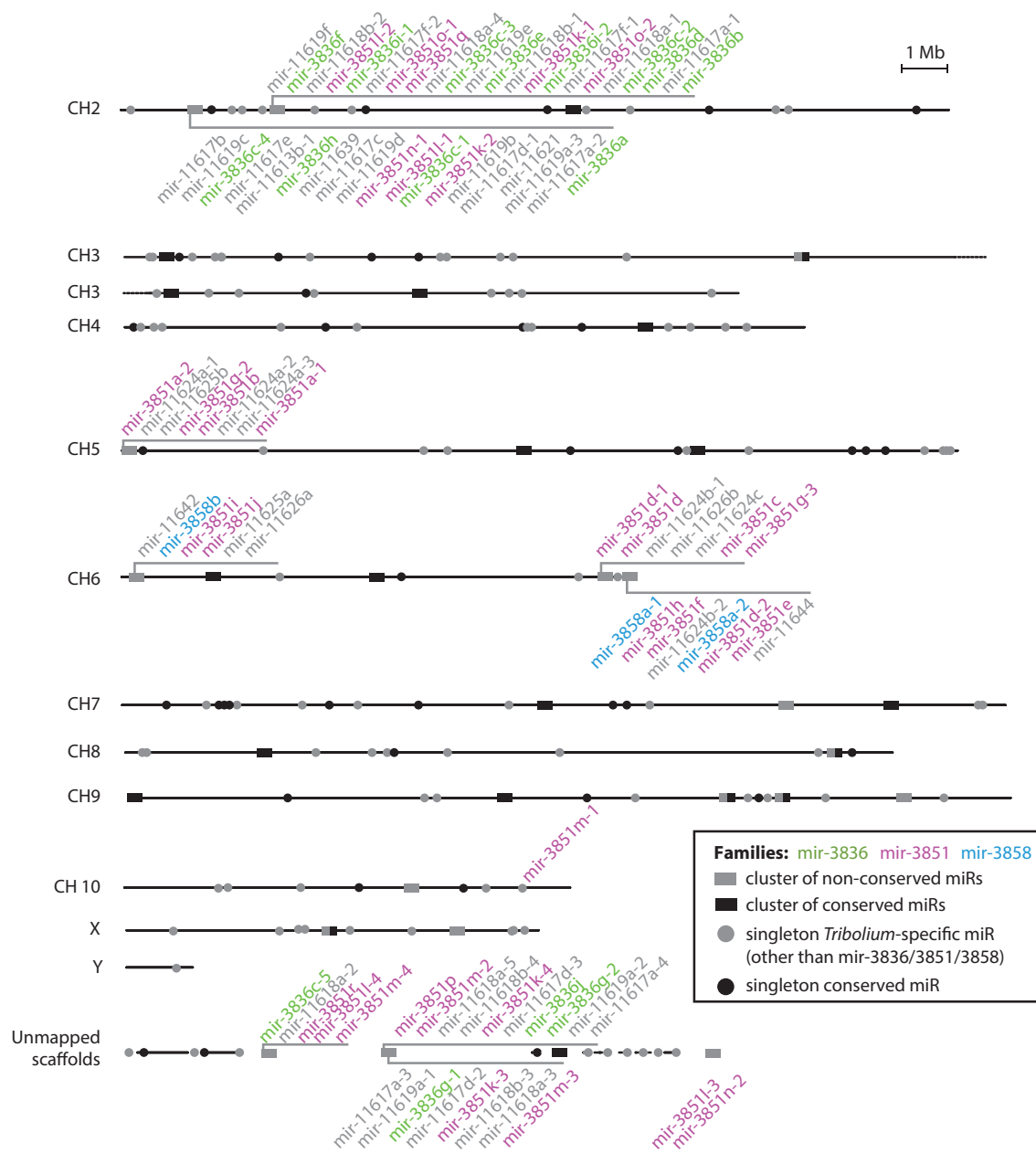

Figure 1. Genomic organization of known and novel microRNAs in T. castaneum. Horizontal lines represent the assembled chromosomes and the unmapped scaffolds of the $T$. castaneum genome assembly (r4.0), on which microRNA genes are found. MicroRNA-encoding loci are marked, and microRNAs within a 10-kb distance from each other are shown together as a cluster. Clusters encoding members of widespread Tribolium-specific microRNA families are labeled (see main text), and colors show paralogous family relationships.

the transcriptionally inactive 0-5-h embryos, with highly abundant piRNAs. In contrast, D. melanogaster and D. virilis oocyte small RNA profiles display two prominent peaks corresponding to maternally provided microRNAs and piRNAs. MicroRNA read count normalization according to the endogenous and spiked-in references independently and consistently shows $\sim 0.2 \mathrm{fmol}$ of microRNAs ( $\sim 120$ million microRNA molecules) per egg in Tribolium, and approximately four times higher microRNA content in Drosophila (Fig. 3B,C). This difference in microRNA abundance between the fly and beetle is not sufficient to explain the difference in the microRNA/piRNA ratios in the two taxa (see Discussion).

We next assessed the most abundant maternally deposited microRNAs in Tribolium and compared these to fruit flies (Fig. 3D; Supplemental Table 2). The data show that the conserved microRNA families bantam, mir-275, mir-305, mir-14, mir-184, mir-995, mir-2/11/13, mir-92/310, mir-279, and mir-9 represent the most highly maternally loaded microRNAs in the insect oocytes. Clustered microRNAs are usually co-expressed, consistent with previous findings (Baskerville and Bartel 2005; Ruby et al.
2007; Ryazansky et al. 2011). A notable example of divergence of microRNA maternal deposition is the mir-100/let7/mir-125 cluster, members of which are loaded in Tribolium but not in Drosophila. In addition, the most abundant microRNAs in the beetle oocyte belong to two clusters: mir-279e 2944c and mir3889 3843. These are diverged homologs of the Drosophila mir-309 6 and mir-994 318 (Ninova et al. 2014), whose maternal deposition in Drosophila is modest. The maternal microRNA complement of T. castaneum also contains a number of lineage-specific microRNAs, including the members of the massively duplicated 40 miRNA gene cluster on the X Chromosome, albeit at lower concentrations (Supplemental Table 2).

MicroRNA 3' ends are known to be subject to various modifications in different model systems, including ligation of additional nucleotides to the $3^{\prime}$ end of the mature product (Ha and Kim 2014). A recent study reported that up to $30 \%$ of microRNAs in the oocytes and early embryos of D. melanogaster and deuterostomes are $3^{\prime}$ end-modified, and in D. melanogaster this modification was suggested to be involved in maternal microRNA clearance (Lee et al. 2014). We addressed the presence and developmental dynamics of microRNA 3'-end nucleotide additions in $T$. castaneum deep-sequencing data, together with a D. virilis developmental time series previously generated by our group for comparison (Ninova et al. 2014). Because we can only detect 3 ' end addition of nucleotides different from the ones at the genomic position immediately after the microRNA 3' end cleavage site, our estimates of the extent of microRNA modifications in insect oocytes are likely to be conservative. Results showed at least $20 \%$ of the maternally deposited microRNAs in T. castaneum are 3 ' end-modified (Fig. 4A), which is significantly higher than later times or in other tissues, consistent with the previous observations in D. melanogaster (Lee et al. 2014). The majority of nontemplate $3^{\prime}$ end additions are between 1 and 3 nt in length (Fig. 4B) and are almost exclusively adenosines (Fig. 4C). The proportion of reads with modified ends varies between different microRNAs and is poorly correlated with expression level—while some highly abundant microRNAs are not significantly modified, several display a particularly high ratio between their modified and unmodified forms in oocytes (Fig. 4D). These observations are again consistent with those in D. melanogaster (Lee et al. 2014). Notably, among the conserved maternally loaded microRNAs in Drosophila and Tribolium, products of the same families (e.g., miR-184-3p, miR-279-3p, miR-9-3p, miR-92/310-313-3p) are heavily modified. Thus, the process of oligoadenylation of specific maternally deposited microRNAs is conserved between the two insect taxa. 

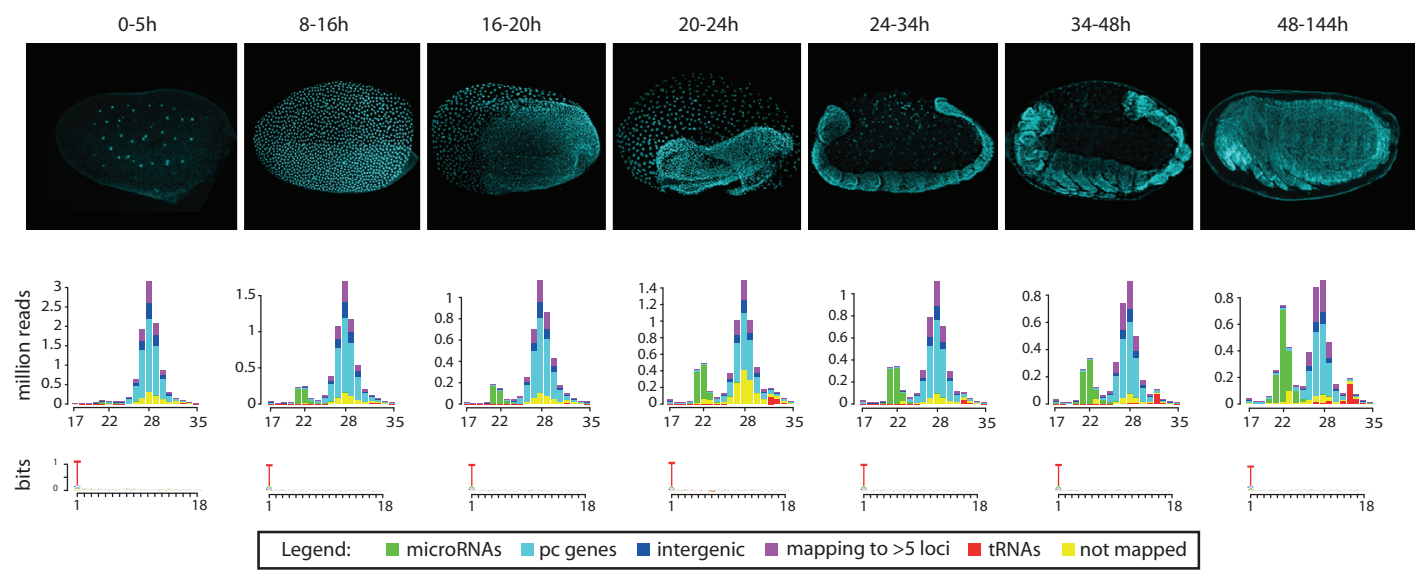

Figure 2. Small RNA types and size distributions throughout T. castaneum development. Histograms show the small RNA size and count distribution in each small RNA library (DAPI-stained representative images for the corresponding time intervals are shown in the top panels). Different colors reflect read mapping status: (yellow) not mapped, (purple) mapping to >5 loci, or (red) mapping to at least one tRNA, (green) microRNA, (cyan) protein coding (pc) gene, or (blue) intergenic regions. Sequence logos show the nucleotide bias for the first 18 positions of reads.

\section{Developmental expression of conserved and nonconserved microRNAs}

We next investigated the temporal dynamics of microRNA expression throughout beetle development. MicroRNA read counts from the different small RNA sequencing data sets are shown in Supplemental Table 2. Figure 5A shows the correlations of microRNA expression profiles between the different developmental stages of Tribolium in an all-versus-all manner. As expected, the microRNA repertoire in oocytes and 0-5-h embryos is highly similar, as zygotic expression is not active during the initial cleavage divisions. The similarity between pre-ZT and blastoderm embryos (8-16 h), however, is significantly lower, indicating a shift in the microRNA expression profile upon activation of zygotic transcription. Subsequently, the correlation of microRNA expression is the highest between neighboring stages, and similarity decreases with increasing developmental distance. Thus, developmental transitions in $T$. castaneum are accompanied by shifts in the global microRNA profiles.

To gain further insight into the diversity and dynamics of the microRNA complement throughout beetle embryogenesis, we assessed the normalized expression levels of the annotated T. castaneum microRNAs at each developmental interval. Heat maps in Figure 5B show the levels of individual microRNAs grouped by conservation in other species. Consistent with previous notions, conserved microRNAs are generally more highly expressed (Ruby et al. 2007; Liang and Li 2009; Roux et al. 2012; Meunier et al. 2013). We detect virtually all conserved microRNAs during at least one stage of development, with the vast majority displaying their highest levels in the late embryo during morphogenesis and organogenesis (48 h-6 d). The most strongly expressed microRNAs during the early and intermediate stages of embryogenesis derive from three clusters, mir-9d 3791, mir-3889 3843, and mir-279e 2944c (Fig. 5B, black arrowheads). These clusters encode a combination of conserved and Triboliumspecific microRNAs (Fig. 5C); clusters encoding homologs of the conserved mir-9, mir-2944, mir-279, mir-3791, and mir-309 families are found in other insect lineages and represent one of the most extreme examples of microRNA gain, loss, duplication, and rearrangement reported to date (Ninova et al. 2014). Previous studies by us and others have shown that members of one of these clusters (mir-309 6) in Drosophila are strongly up-regulated in the early fly blastoderm (Biemar et al. 2005; Ninova et al. 2014), and that members of the homologous clusters in A. mellifera (mir-3478 318) and mosquitoes (mir-309 286) are also highly expressed in early embryos (Zondag et al. 2012; Hu et al. 2014). In addition, the mosquito-specific mir-2941 2946 cluster, whose 3' mature sequences have the same seed as miR-3889-3p in T. castaneum, are the most abundant microRNAs in the early stages of development (Hu et al. 2014). We detect nascent primary transcripts of mir-9d 3791 and mir-3889 3843 in the early blastoderm nuclei of $T$. castaneum by in situ hybridization (Supplemental Fig. $4 \mathrm{~A})$. Taken together, these data suggest that, despite multiple rearrangements, the early zygotic onset of expression of these groups of microRNAs is conserved. Interestingly, we detect nascent mir$9 d \sim 3791$ transcripts much later in development, in serosal nuclei, suggesting a novel role of members of this cluster (Supplemental Fig. 4B).

While the levels of Tribolium-specific microRNAs are low during most developmental stages, the majority display a coordinated sharp increase in expression at the undifferentiated blastoderm stage (8-16 h) (Fig. 5B,D). Without exception, the blastodermspecific pool of nonconserved microRNAs belong to the multicopy novel clusters encoding divergent members of the mir-3851, mir3836, and other Tribolium-specific microRNA families described above (Fig. 5B, gray arrowheads; also see Fig. 1). The expression patterns of these microRNA clusters suggest that they are up-regulated for a discrete period of time, immediately following the onset of zygotic transcription in the blastoderm, and are rapidly extinguished shortly afterward. To test this, we assessed the expression of putative nascent transcripts corresponding to selected highly expressed nonconserved microRNA clusters by in situ hybridization (Fig. 5E). Due to the high sequence similarity between some regions, we expect that a subset of probes would cross-hybridize and detect transcription from more than one locus, and indeed this is the case for the mir-3851a-1 region (Fig. 5E, third panel). The data show that the multicopy microRNA clusters are ubiquitously expressed in all blastoderm nuclei, but surprisingly, their expression is limited to a very narrow time interval from the 8th-9th to the 11 th cleavage division.

\section{Genome Research}


A

D. melanogaster

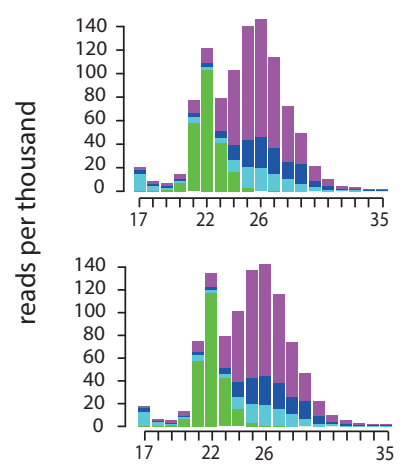

D. virilis
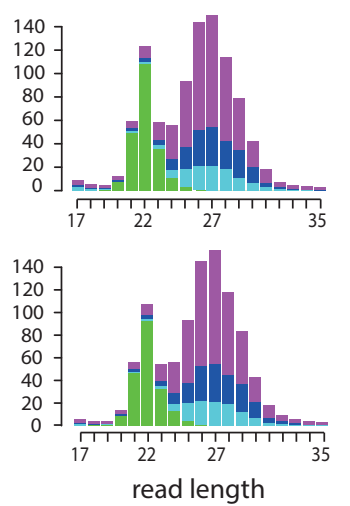

D

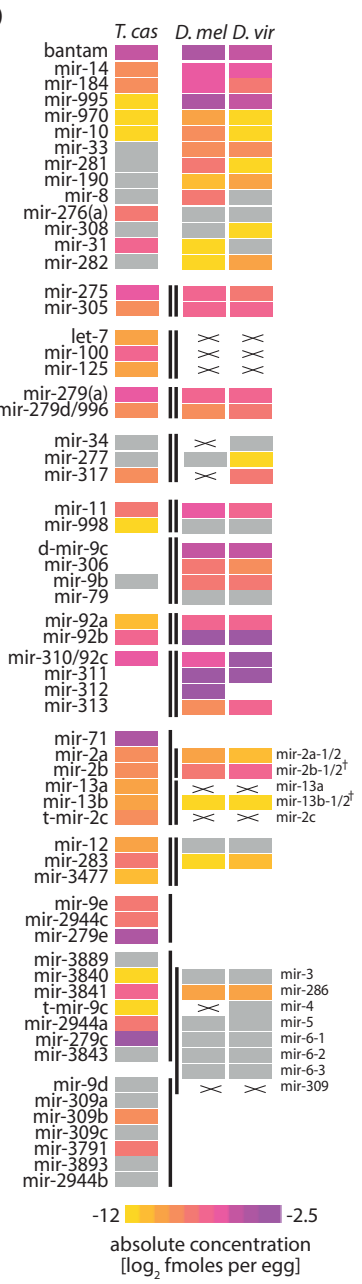

Figure 3. Quantities and conservation of maternally deposited microRNAs. (A) Read size and count distributions of small RNA sequencing libraries from oocytes in the three species (two biological replicates per species). Reads are colored depending on their mapping position to microRNAs (green), proteincoding (pc) (cyan), intergenic regions (blue), or multiple $(>5)$ sites in the genome (purple). (B) Absolute abundance of total microRNAs as determined by deep-sequencing read counts relative to spike-in. (C) Absolute abundance of total microRNAs as determined by deep-sequencing read counts relative to miR-184-3p. In $B$ and C, quantification from deep-sequencing analysis was performed using one and two allowed mismatches between read and genome. (D) Heat map showing the estimated concentrations of conserved microRNAs or clusters of microRNAs between Drosophilids and T. castaneum. MicroRNAs below an arbitrary threshold of $0.0002 \mathrm{fmol} / \mathrm{cell}$ are shown in gray. Lines mark clustered microRNAs. Crosses mark conserved microRNAs that are not expressed, while blank spaces denote absence of a given homolog from the corresponding cluster in a given species. MicroRNAs are aligned by homology; note that the mir-3 309 cluster of Drosophila has three homologs in Tribolium. $\left(^{\dagger}\right)$ mir-2 cluster in Drosophila has split.

\section{Abundant early expressed microRNAs target maternally loaded and zygotically down-regulated genes}

Very early expressed microRNAs have been shown to play a role in the clearance of maternally deposited transcripts in both Drosophila and zebrafish (Giraldez et al. 2006; Bushati et al. 2008). However, the microRNAs involved in this process in the two species (mir-309 6 and mir-430 clusters, respectively), are not homologous, and microRNA-dependent maternal transcript clearance is thought to be a convergent phenomenon. Nonetheless, a common feature is that in both taxa these microRNAs are encoded in large clusters that have undergone multiple duplications and diversification. The most highly expressed microRNAs in the early embryo of $T$. castaneum are also encoded in large clusters, including mir-9d 3791 (homologous to the fruit fly mir-309 6 cluster [Ninova et al. 2014]), mir-279e 2944c, and mir-3889 3843 (Fig. $5 \mathrm{~B}, \mathrm{C})$, as well as other large species-specific clusters discussed above
(Figs. 1, 5B). Thus, we asked whether these or other T. castaneum microRNAs might be involved in maternal transcript clearance.

To determine the maternally deposited mRNA complement, and its fate after zygotic genome activation, we used RNA sequencing to estimate protein-coding transcript levels in unfertilized oocytes, early blastoderm embryos (8-16 h), embryos at the stage of blastoderm differentiation, gastrulation, and serosal closure (16-24 h), and embryos at the stage of germband elongation and segmentation ( $24-48 \mathrm{~h}$ ). The resulting $\sim 300$ million paired-end reads were mapped against the $T$. castaneum genome and transcriptome, detecting at least one fragment for 15,221 out of the 16,503 annotated protein-coding genes (92\%). Gene expression levels were highly similar between replicates $(r>0.96)$, and differential expression analyses show that a large number of transcripts significantly change their abundance between different intervals (Fig. 6A; Supplemental Fig. 5). In particular, we find a large number of transcripts that are highly up-regulated between embryonic 
A

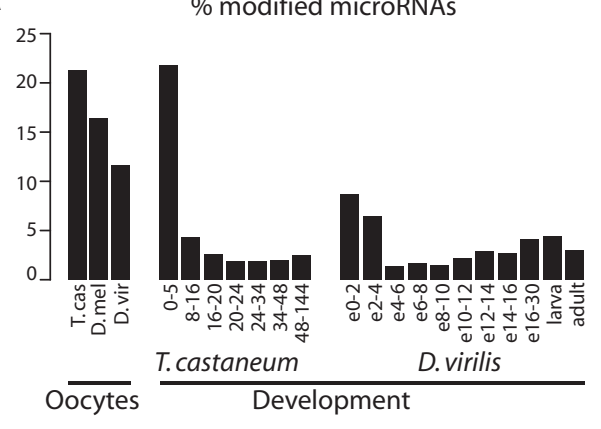

B

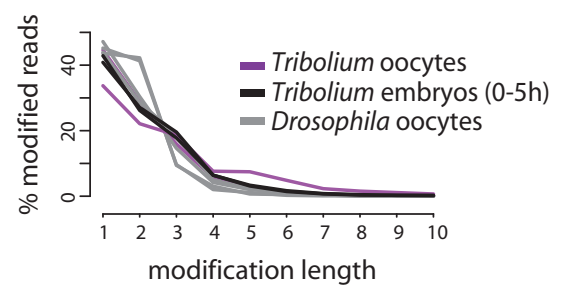

C

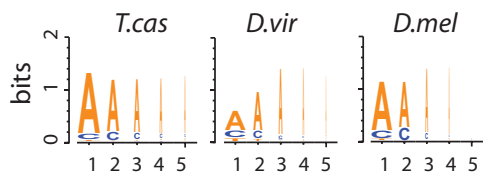

D
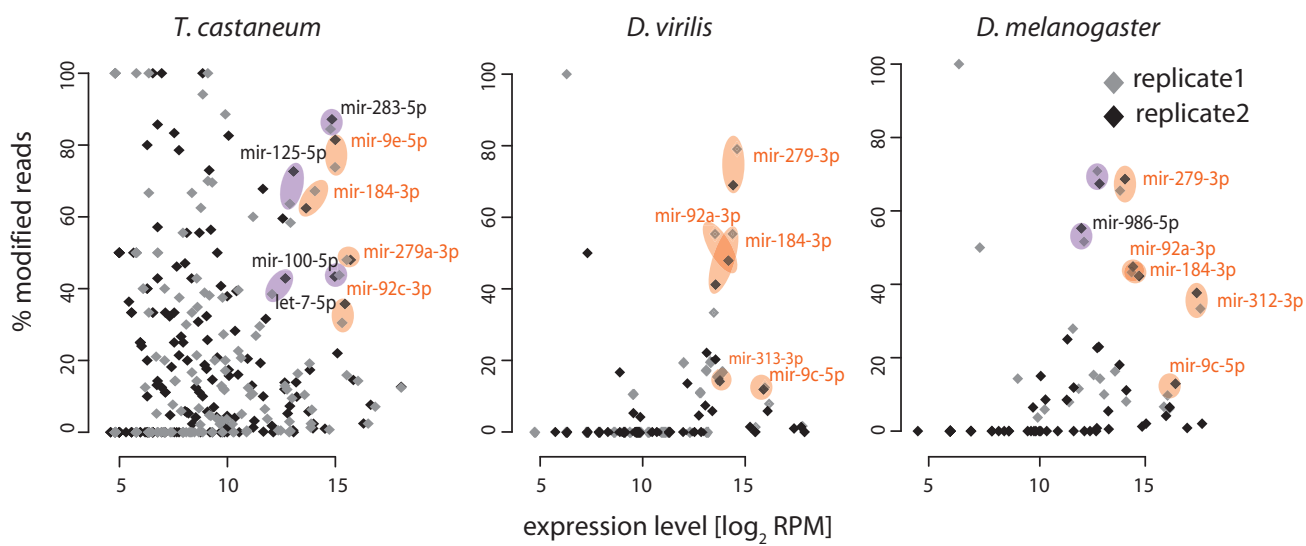

Figure 4. Nontemplated $3^{\prime}$-end nucleotides in oocyte microRNAs. (A) Proportion of microRNAs with nontemplate $3^{\prime}$-end nucleotide addition in oocytes and developmental small RNA libraries of $T$. castaneum and Drosophila. (B) Length distribution of nontemplate $3^{\prime}$-end nucleotide additions. (C) Sequence logos showing the most frequent nucleotides in the first five positions of the $3^{\prime}$-end nontemplated nucleotide additions in the three species. (D) Scatter plots showing the proportion of reads with nontemplate $3^{\prime}$-end additions as a function of the total read counts (modified and nonmodified) per million for each microRNA. Results of different replicates for each species are shown separately. Example singleton microRNAs or microRNAs from clusters that are conservatively deposited in the egg are highlighted in orange, and highly deposited and strongly modified microRNAs specific for a given species are highlighted in purple.

development and oocytes, likely reflecting developmental processes activated in the early embryo. A substantial number of transcripts also display a smaller yet significant negative change in their levels with the progression of embryogenesis, indicating maternal transcript clearance. We predicted the putative microRNA targets of the T. castaneum transcripts by two different microRNA target prediction approaches-detection of canonical microRNA-target binding sites (Bartel 2009), and using the miRanda algorithm, which takes into account sequence complementarity and RNA-RNA duplex free energy (Enright et al. 2003). These methods resulted in 12,565 and 12,660 predicted microRNA targets among the 13,412 genes with available 3' UTR annotations, respectively, with 144,579 individual microRNAtarget pairs overlapping between the two sets, and 276,412 and 241,723 pairs unique for each set. We then calculated whether the targets of individual mature microRNAs are enriched among the protein-coding transcripts that are significantly (more than twofold) down-, up-, or not regulated between oocytes and embryos. Despite the poor overlap of individual target sites between the target prediction methods, the overall trends in microRNA target enrichment are highly consistent. Distributions of the hypergeometric $P$-values of target enrichment based on canonical interac- tions are shown in Figure 6B, and the complete data sets for both target prediction algorithms, as well as analyses performed using an alternative differential expression algorithm, are available as Supplemental Table 3. The significance of microRNA target site enrichment within zygotically up- and down-regulated genes was additionally assessed by permutation tests, yielding similar results (Supplemental Table 3). The data show that genes that are downregulated between oocytes and embryos, particularly in the early blastoderm (8-16 h), are strongly enriched in targets of a specific set of microRNAs. In general, these microRNAs do not target a significant proportion of genes that are up-regulated or that maintain their expression. We further assessed the expression levels, sequence, genomic localization, and evolutionary relationships of the microRNAs that specifically target zygotically down-regulated genes but not up-regulated genes (enrichment of targets in the down-regulated set with $P<0.001$ after Bonferroni correction). First, we note that this set of microRNAs consists of multiple paralogs from a small number of families, inferred by manual inspection of hairpin multiple sequence alignments (Fig. 6B labels; Supplemental Fig. 2). Altogether, this set of microRNAs includes 15 of the 16 members of the mir-279e 2944c, mir-3889 3843, and mir-9d 3791 clusters, as well as novel and previously

\section{Genome Research}

www.genome.org 


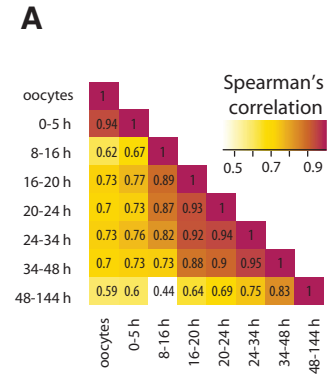

\section{C}

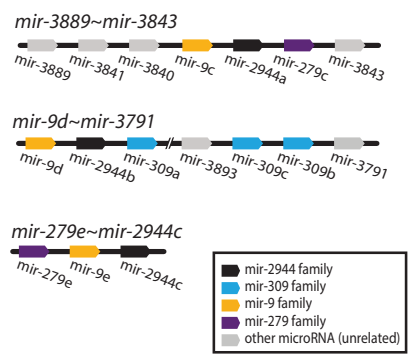

D
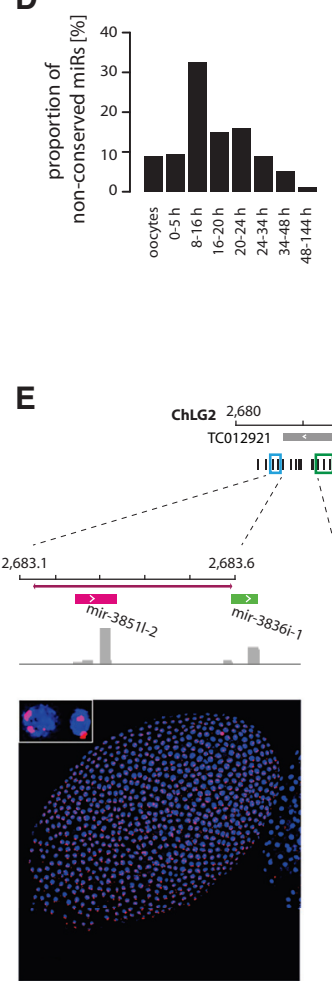

B
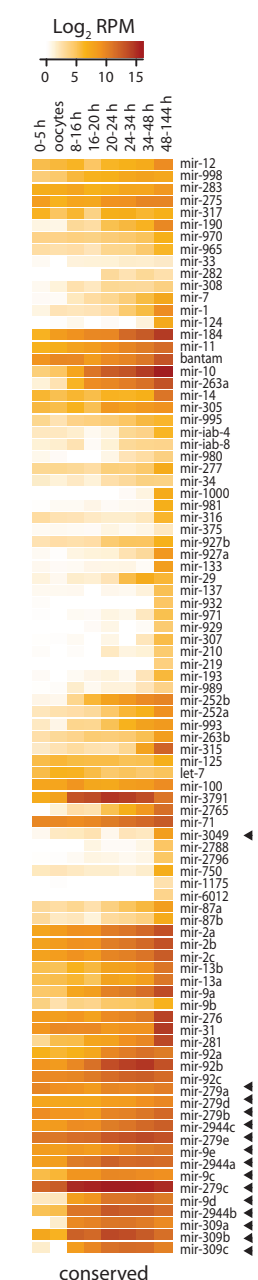

conserved

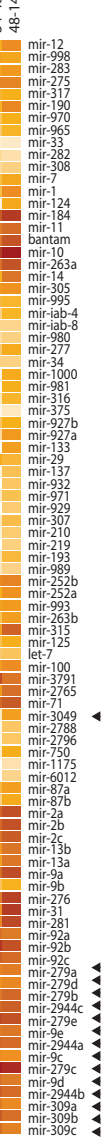

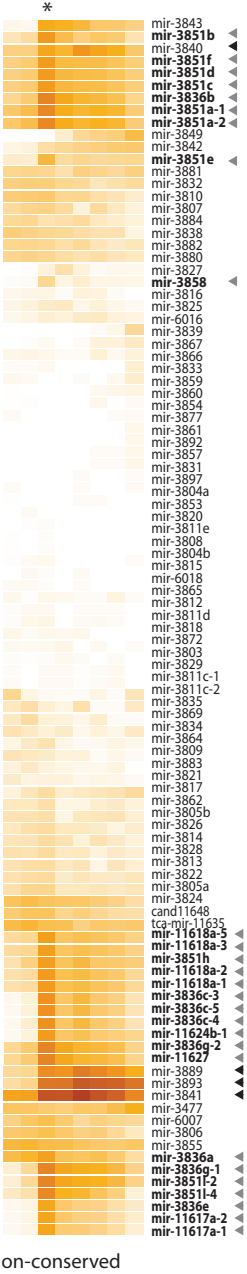

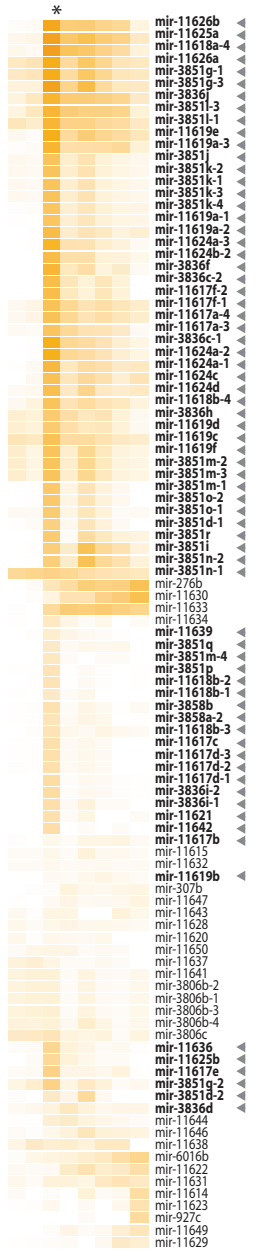
ChLG5 238 kb Unmapped 58 20, 230 kb ||I| ||⿴囗十) IIII | ||

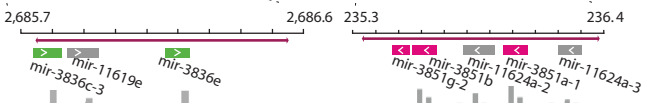

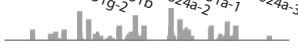
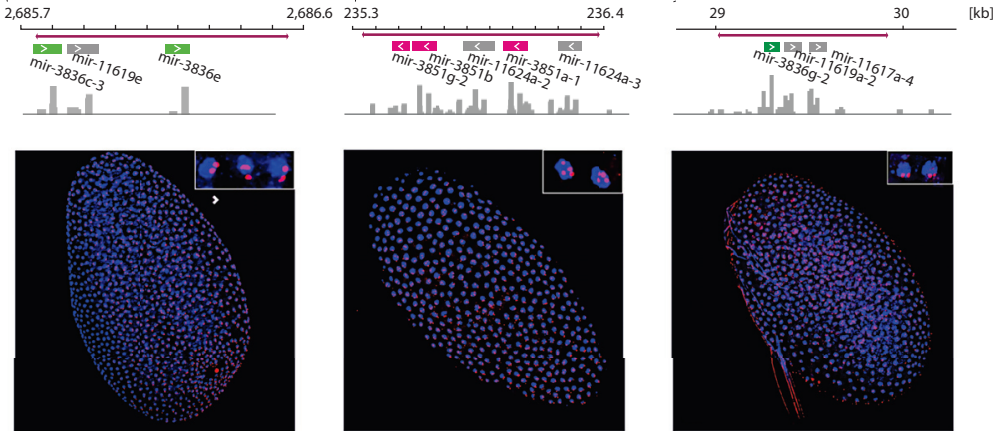

Figure 5. Developmental expression of conserved and nonconserved microRNAs throughout $T$. castaneum development. $(A)$ Heat maps representing Spearman's correlation values for all-versus-all comparisons of the microRNA expression levels in oocytes and embryonic intervals of 0-5, 8-16, 16-20, 20-24, 24-34, 34-48, and 48-144 h. (B) Heat maps showing the normalized expression levels (reads per million) of conserved and species-specific microRNAs in T. castaneum oocytes and developmental intervals. Gray arrowheads indicate microRNAs from the mir-3851 and mir-3836 families and other microRNAs clustered in the same loci, as in Figure 1. Black arrowheads mark members of the mir-279e 2944c, mir-3889 3843, and mir-9d 3791 clusters. (C) Diagram of the genomic organization of the T. castaneum mir-279e 2944c, mir-3889 mir-3843, and mir-9d 3791 clusters. MicroRNAs are color-coded based on sequence homology. (D) Relative proportion of Tribolium-specific microRNA reads at different developmental stages. (E) Spatial expression of Tribolium-specific microRNA clusters detected by in situ hybridization. Top diagrams indicate the genomic regions encoding Tribolium-specific microRNAs used for antisense DIG-labeled RNA probe design, with probe regions in colored boxes. MicroRNA genes are black or color-coded based on homology to mir-3851 (magenta) and mir-3836 (green). Histograms represent coverage tracks generated with IGV. (Bottom) Confocal images of $T$. castaneum blastoderm embryos showing nascent microRNA transcripts (red); blue represents DAPI nuclear staining. 
A

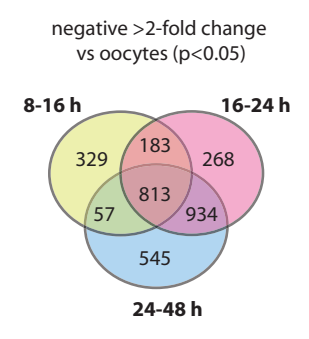

positive $>2$-fold change vs oocytes $(p<0.05)$

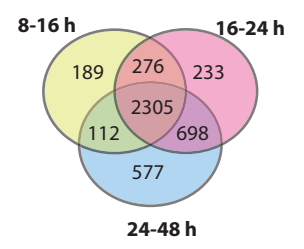

C

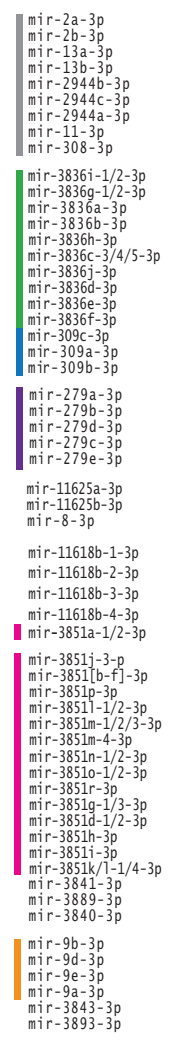

B

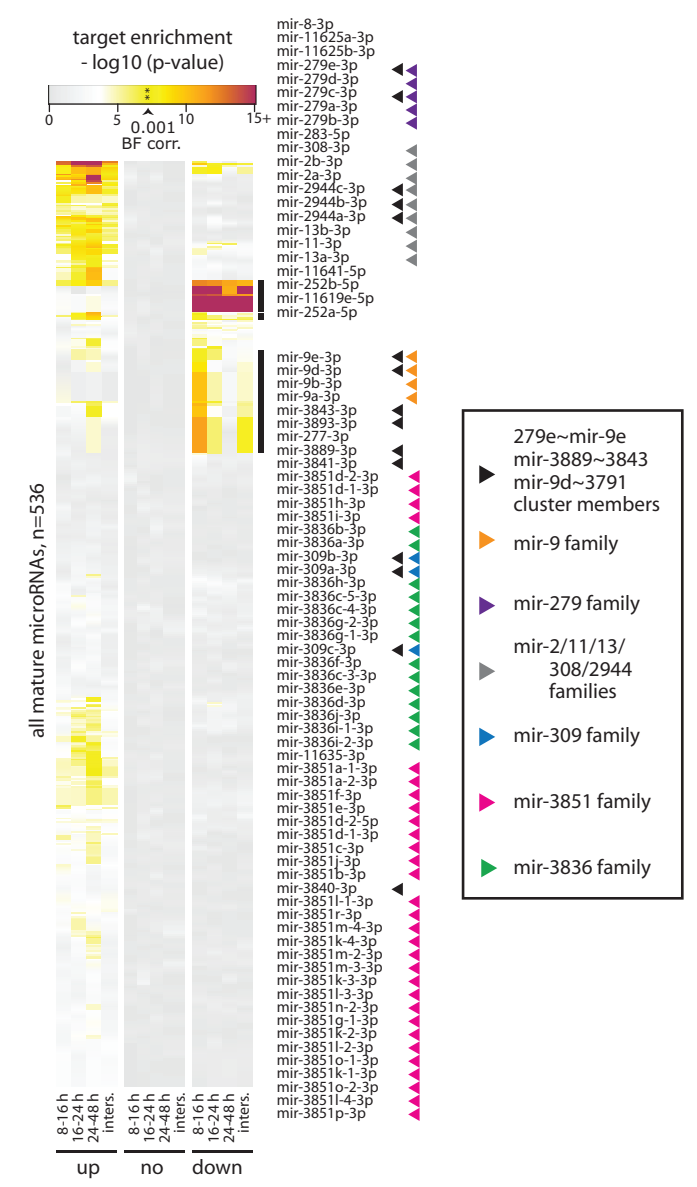

D

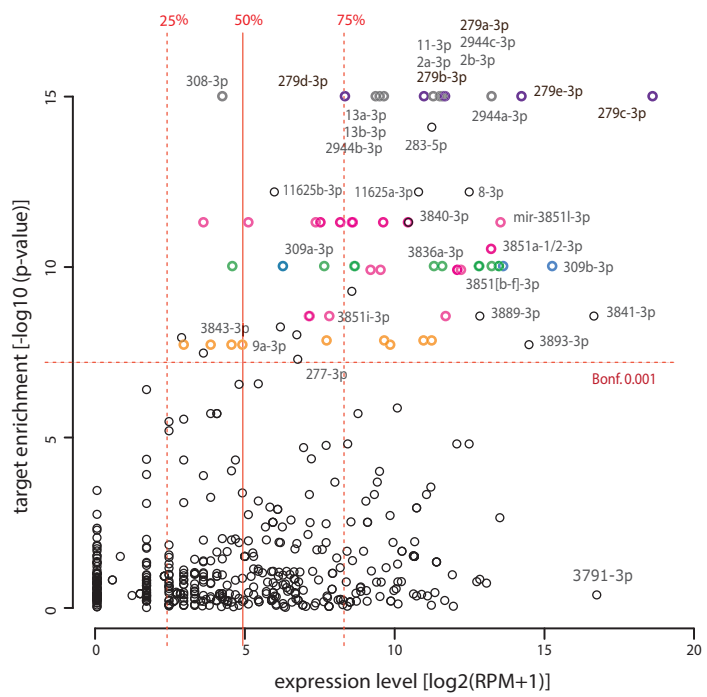

Figure 6. MicroRNA targeting of maternally deposited transcripts in T. castaneum oocytes and developing embryos. (A) Venn diagrams showing the number and overlap between greater than twofold up- and down-regulated transcripts $(P<0.05)$ between $T$. castaneum oocytes and the three embryonic time intervals (8-16, 16-24, and 24-48 h). (B) Hypergeometric $P$-value for mature microRNA targets enrichment predicted based on canonical site interactions among genes down-regulated ("down"), up-regulated ("up"), and not regulated ("none") from oocytes to 8-16, 16-24, and 24-48 h embryos. "Inters" denotes the intersection of the three individual sets. The two groups of microRNAs with enrichment $P$-value $<0.001$ (Bonferroni corrected) in the downregulated transcripts are expanded and labeled (right). Arrowheads indicate microRNA family and clustering. The values underlying the heat map representation and full data are available as Supplemental Table 3. (C) Alignments of mature sequences of major seed families with highly enriched targets in the zygotically down-regulated gene set. Side bars indicate microRNA families color-coded as in B. Shading indicates $100 \%$ base identity at a given position. MicroRNAs with identical sequences are collapsed on a single line. $(D)$ Relationship of microRNA expression and target enrichment in the down-regulated gene set in the 8-16 h embryo. Vertical lines show the median, upper, and lower quantile values, and horizontal line shows the $0.001 P$-value after Bonferroni correction threshold. Points corresponding to the microRNAs outlined in $B$ and $C$ are color-coded accordingly. Selected microRNAs are labeled.

\section{Genome Research}

www.genome.org 
annotated members of the mir-3851 and mir-3836 families, which are uniquely up-regulated upon the activation of zygotic transcription. Sequence comparisons further showed that many microRNAs that lack overall sequence similarity have identical 6-,7-, or 8-mer seed regions (herein referred to as "seed families") (Fig. 6C), and thus have highly similar predicted canonical target sets. Major seed families include AGUACG (3p arms of mir-3851b-q, mir3889, mir-3840, and mir-3841), AGUACA (3p arm of mir-3851a and mir-11618b), CACUGG (3p arms of mir-3836 and mir-309/3 families), AUCACA (3p arms of mir-2944, mir-2/13, mir-11, and mir-308; complementary to the K-box motif) (Lai et al. 1998), UAAAGC (3p arms of mir-9, mir-3893, and mir-3843; complementary to the Brd-box motif) (Leviten et al. 1997); GACUAG (3p arms of miR-279a to e), and AAUACU ( $3 p$ arms of mir- 8 and novel microRNAs mir-11625a/b). In addition, zygotically down-regulated genes are also enriched in target sites of miR-283-3p, miR-252-3p, and miR-277-3p. Sylamer analysis (van Dongen et al. 2008) showed that of all possible 6-mer nucleotide words, motifs complementary to the seed regions of microRNAs from the above families are among the most highly and significantly enriched motifs in the 3' UTR of the zygotically down-regulated genes (Supplemental Table 4).

Assessment of microRNA expression levels in the context of their targeting properties showed that the vast majority of microRNAs targeting down-regulated genes in the blastoderm are among the most highly expressed microRNAs at that stage (Fig. 6D). The reciprocity between specific microRNA up-regulation and the down-regulation of their targets in the early blastoderm strongly suggests that these microRNAs are involved in maternal transcript clearance in $T$. castaneum. We also note that a different set of highly expressed and conserved microRNAs, including miR-9-5p, mir-263, and mir-276, target a substantial fraction of genes up-regulated during embryogenesis, reflecting a likely role for these microRNAs in other developmental processes (Supplemental Table 3).

Previous findings in $D$. melanogaster showed that highly expressed members of the mir-309 6 cluster in the early embryo, including mir-9/4/79, mir-5/6/2944, mir-3/309, and mir-279/286, are involved in maternal transcript turnover (Bushati et al. 2008). Our results suggest that these microRNA families have a conserved role in maternally deposited transcript regulation in the early embryo of holometabolous insects. Furthermore, we identify several additional, Tribolium-specific microRNA families involved in the process, including mir-3889, mir-3840, mir-3841, and mir-3851 families. We therefore suggest that the microRNA repertoire involved in maternal transcript clearance has diverged.

\section{Discussion}

\section{Abundance and modifications of maternally deposited microRNAs}

Oocyte maturation is accompanied by the deposition of a large number of protein and RNA factors, including small RNAs from the microRNA and piRNA classes. In Drosophila, maternal piRNA deposition is required for the inheritance of transposon defense (Brennecke et al. 2008). The role of maternally provided microRNAs, on the other hand, is not well understood: microRNAs can be detected in Drosophila oocytes, but it is unclear whether these are products generated at earlier stages of gonadal development, or if their deposition is required for subsequent events in embryonic development. For instance, genetic knockout of the maternally provided mir-310 mir-313 cluster in Drosophila does not result in developmental defects (Tsurudome et al. 2010;
Pancratov et al. 2013). MicroRNAs are not present at high levels in small RNA libraries of zebrafish, Xenopus, and mouse oocytes, and the roles of maternally deposited microRNAs in these species are not well understood (Chen et al. 2005; Watanabe et al. 2005; Ohnishi et al. 2010; Lee et al. 2014).

As in vertebrates, the cloning frequency of microRNAs in oocytes of Tribolium is very low compared to subsequent developmental stages. However, absolute quantification of T. castaneum maternally deposited microRNAs shows a concentration of $\sim 0.2$ fmol per oocyte, which is commensurate with previous estimates of microRNA copy numbers in mammalian cells (Bissels et al. 2009) and likely reflects physiologically relevant levels. Thus, the underrepresentation of microRNAs in the T. castaneum oocyte libraries is not due to absence of maternal loading but to very high levels of piRNAs. RNA sequencing shows high levels of transposon activity in the Tribolium embryo, and we speculate that the high abundance of piRNAs is related to this observation (M Ninova, S Griffiths-Jones, M Ronshaugen, in prep.).

The metabolism of maternally provided microRNAs is not well understood. It was recently demonstrated that a substantial proportion of the microRNA complement in oocytes is 3 '-end adenylated post-transcriptionally. Since this modification enhances microRNA degradation, it provides a plausible mechanism to control clearance of maternally loaded microRNAs (Lee et al. 2014). Consistent with this possibility, we detect high levels of 3 '-end oligoadenylated microRNAs in Tribolium and Drosophila oocytes. Interestingly, only specific mature microRNAs are modified at high levels, but this is not obviously determined by microRNA sequence (Lee et al. 2014). Our data demonstrate that the specificity of microRNA adenylation in oocytes is similar for abundant microRNA orthologs between Drosophila and Triboliium, suggesting that the mechanism that regulates this process is conserved among holometabolous insects, across at least $\sim 300$ million years of evolution. Further work is required to elucidate the molecular basis and developmental effects of this process.

\section{MicroRNA-mediated maternal transcript clearance in $T$. castaneum}

One of the earliest events during animal development is the degradation of maternally deposited transcripts in the egg and the activation of the zygotic genome-a process termed the maternal-to-zygotic transition (MZT) (for review, see Tadros and Lipshitz 2009). MicroRNAs have been implicated in the MZT of both invertebrates and vertebrates. However, the data suggest that involvement of microRNAs in this function has evolved convergently in the two clades (Bushati et al. 2008). In zebrafish, among the first zygotically expressed transcripts is a large cluster encoding over 50 mir- 430 paralogs, which target a significant fraction of the maternally provided mRNAs (Giraldez et al. 2006). In Drosophila, a similar role was proposed for the early expressed mir-309 6 cluster (Bushati et al. 2008). Genetic knockout of the mir-309 6 cluster results in the delayed degradation of a number of maternally provided mRNAs but does not cause significant embryonic defects (Bushati et al. 2008). We speculate that members of the mir-309 6 cluster are not the only microRNAs involved in maternal transcript regulation in Drosophila, as other microRNAs from the same seed families-including miR-2/11/13-3p, miR279/996-3p, and mir-9 paralogs—are present in the early embryo.

We have identified a number of conserved and species-specific microRNAs in T. castaneum that are highly expressed in the early embryo and target a significant fraction of down-regulated maternal transcripts during the MZT. Thus, the data suggest that 
microRNAs are involved in the degradation of maternally deposited transcripts in this lineage. The conserved microRNAs involved in maternal transcript down-regulation in the blastoderm of the flour beetle include homologs of the Drosophila mir-309 6 cluster members and other microRNAs with identical seeds. Taken together, the data suggest that microRNA-mediated maternal transcript degradation by the $3 p$ mature arms of microRNAs from the seed families AUCACA (mir-2944/5/6, mir-2/13 and mir-11), UAAAGC (mir-9/79/4 family), and CACUGG (mir-309/3 family) is a conserved feature in holometabolous insects. mir-279 family members are very highly expressed in the early embryo and show one of the strongest target enrichment values among the down-regulated transcripts in the early embryo. In addition, we observed high expression levels and target site enrichment in the zygotically downregulated genes targeted by mir- 8 and mir-283, and to a lesser extent, mir-277 and mir-252. The roles of these microRNAs in maternal transcript clearance in Drosophila have not been previously addressed; further studies are required to determine whether the involvement of these deeply conserved microRNAs in the MZT is conserved in insects or represents a Tribolium-specific co-option.

Several $T$. castaneum-specific microRNAs also target a significant fraction of the maternally deposited transcripts that decrease at the blastoderm stage. These include the multicopy microRNA families mir-3836 and mir-3851 and four hairpins encoded in the mir-3889 3843 and mir-9d 3791 clusters: mir-3889, mir-3840, and mir-3841, and mir-3893. Notably, 3p products corresponding to the seed families of mir-3851a (AGUACA) and mir-3851b to q, mir-3889, mir-3840, and mir-3841 (AGUACG) are not found in Drosophila, suggesting that these microRNA-target interactions are a diverged feature between these insect lineages.

Taken together with previous studies in Drosophila, findings in $T$. castaneum suggest that microRNA-mediated maternal transcript degradation is a conserved mechanism in holometabolous insects, but the precise microRNAs participating in this process differ somewhat between species. MicroRNAs involved in maternal transcript clearance in vertebrates (Giraldez et al. 2006; Lund et al. 2009) have no sequence similarity or common seed motifs with any of the insect microRNAs, illustrating the likelihood of convergence in this process (see below). Nevertheless, comparisons of the microRNAs in the MZT in different organisms reveal the common phenomenon of large, fast-evolving microRNA polycistrons involved in this process.

\section{Dynamic evolution of early expressed microRNAs}

Our analysis of the genomic positions, sequences, and targeting properties of the early expressed $T$. castaneum-specific microRNAs reveal complex relationships. The mir-3851 and mir-3836 families, and novel mir- 8 seed family members, are found in large and diverse clusters located in multiple genomic positions. These microRNAs are not colocalized with any conserved microRNAs, but other members from these seed families, such as mir-309, have deeper evolutionary origins and are clustered with other conserved microRNAs. MicroRNA hairpins are short, and thus any putative fast-evolving sequences can diverge to the point at which they cannot be confidently identified as homologs. On the other hand, the formation of microRNAs with identical seeds (microRNA convergence) may be common, as the microRNA seed region is very short, and new hairpins often emerge de novo in animal genomes. Despite their high degree of divergence in terms of encoded hairpin copy number, family, and sequence, the $T$. castaneum-specific microRNA clusters display a very similar temporal expression pattern spanning only a few rounds of cell division after the initiation zygotic transcription. We propose that newly emerged microRNAs with convergent seeds and similar expression patterns to existing microRNAs are more likely to be retained, as they "mimic" the existing microRNA and thus do not cause significant transcriptome perturbations by down-regulating new transcripts. In the light of this hypothesis, one explanation for the origin of the mir-3836 and mir-3851 clusters is that their founding members emerged from random hairpins in early activated regions and subsequently duplicated and diversified. Alternatively, if microRNAs with identical seeds are considered to be highly diverged paralogs, we can speculate that the mir-3836 and mir-3851-encoding clusters, and the three clusters encoding members of the conserved mir-5/6/2944, mir-9/4, mir279/286, and mir-309/3 families, have common origins but have significantly diverged via multiple duplications, rearrangements, and losses (including the acquisition of a mir-8 paralog that rapidly diverged). These scenarios of cluster evolution are not mutually exclusive: It is likely that some seed families are evolutionarily related, while others emerged by convergence. Either way, the evolutionary patterns of the early expressed microRNAs are uniquely dynamic.

Our previous work demonstrated that one characteristic of the early Drosophila embryo is high levels of fast-evolving microRNAs (Ninova et al. 2014). Data from T. castaneum now suggest that the early embryonic expression of fast-evolving and evolutionarily younger microRNAs is not restricted to Drosophila but represents a conserved feature of holometabolous insects. Studies in other organisms have also suggested that early embryogenesis is permissive or robust to evolutionary change in the transcriptome compared to later stages of development: In Drosophila, vertebrates, and plants, the early embryonic transcriptome is, on average, younger, faster evolving, and characterized with higher variation in orthologous gene expression (Domazet-Lošo and Tautz 2010; Kalinka et al. 2010; Quint et al. 2012; Heyn et al. 2014). Even though the underlying causes of this phenomenon are elusive, our results suggest that the apparent flexibility of the molecular networks active in early development also impacts the evolution of the microRNA complement expressed at that stage.

\section{Methods}

\section{Animal husbandry, sample collection, and deep sequencing}

T. castaneum wild-type adults (Michael Akam, University of Cambridge) were reared following a standard protocol (The Beetle Book: http://wwwuser.gwdg.de/ gbucher1/tribolium-castaneumbeetle-book1.pdf) at $25^{\circ} \mathrm{C}$, and wild-type D. melanogaster and $D$. virilis were maintained under standard conditions. Details on embryo collection timing are provided in the Supplemental Text. RNA was extracted using a standard TRIzol protocol. Small RNA and RNA libraries were constructed using the Illumina TruSeq Small RNA Sample Prep and TruSeq Stranded mRNA Sample Prep kit, respectively. Libraries were assessed using the Agilent 2200 TapeStation and sequenced on the Illumina MiSeq (embryonic small RNA sequencing) or the Illumina HiSeq 2000 (oocytes small RNA sequencing and whole-transcriptome sequencing) platforms in the University of Manchester Genomic Technologies facility.

\section{Embryo fixation, immunohistochemistry, and in situ hybridization}

Embryos were dechorionated, fixed, and devitellinated using a standard protocol. Whole-mount fluorescent in situ hybridization

\section{Genome Research}

www.genome.org 
with $\sim 1$ kb-long DIG-labeled antisense RNA probes and antibody staining procedures were performed according to the protocol in Kosman et al. (2004), but omitting the proteinase K treatment step. Primers for RNA probe synthesis templates and antibodies used for detection are listed in Supplemental Table 5. Images were visualized by confocal microscopy on an Olympus FV1000, and image stacks were processed with Fiji (Schindelin et al. 2012).

\section{Small RNA sequencing data analysis and microRNA prediction}

Adapter sequences were trimmed from the T. castaneum small RNA reads using the Cutadapt tool (http://code.google.com/p/ cutadapt/), retaining reads longer than $16 \mathrm{nt}$. Reads were first filtered against $T$. castaneum tRNA genes predicted using tRNAscanSE (v1.3) (Lowe and Eddy 1997), and then mapped to the latest version of the T. castaneum genome assembly (r4.0) using Bowtie (v1.0) (Langmead et al. 2009) with the following parameters: -v 1 -a -best -strata -m 5. Mapped reads were used as input to two independent microRNA discovery methods-mirdeep2 (Friedländer et al. 2008) and an implementation of the method described in Marco et al. (2010). Newly discovered microRNAs were submitted to miRBase (Kozomara and Griffiths-Jones 2014). MicroRNA read counts were calculated, correcting for mapping to multiple locations, and expression was normalized as reads per million mapped to the genome. $D$. melanogaster and $D$. virilis oocyte small RNA libraries were analyzed as previously (Ninova et al. 2014).

For 3' nontemplate end additions, reads that did not map to the genome with 0 mismatches were sequentially trimmed by $1 \mathrm{nt}$ from their $3^{\prime}$ end and remapped. Upon each iteration, perfectly mapping reads were retained. Resulting trimmed sequences corresponding to full-length microRNAs were analyzed using a custom Perl script (see Supplemental Material).

\section{MicroRNA evolutionary conservation}

T. castaneum microRNAs were grouped into families based on best BLASTN hits (-word_size $=4$ ) (Altschul et al. 1990) and manual inspection and editing of the resulting alignments using RALEE (Griffiths-Jones 2005). Curated alignments were used to build covariance models, and these models were searched against the genomes of Dendroctonus ponderosae, D. melanogaster, D. virilis, A. mellifera, and Bombyx mori using INFERNAL (Nawrocki et al. 2009 ) with an $E$-value cutoff of 1 . MicroRNAs with no hits were considered species-specific. Hits scoring below this threshold were added to previous alignments and manually inspected for hairpin folding and homology.

\section{RNA sequencing data analysis and differential expression}

Paired-end transcriptome data were mapped to the T. castaneum genome (r4.0) using TopHat (Trapnell et al. 2009) with default parameters and supplying the currently available protein-coding gene annotations (iBeetle, http://bioinf.uni-greifswald.de/tcas/genes/ annotation/). Gene expression counts were obtained using htseqcount (Anders et al. 2015), and differential gene expression between oocytes, 8-16-, 16-24-, and 24-48-h embryos was assessed using the DESeq R package (Anders and Huber 2010). Gene expression changes with $P$-values smaller than 0.05 after BenjaminiHochberg correction were considered as significant. We further validated these results using the Cuffdiff program from the Cufflinks package (Trapnell et al. 2010) as an alternative approach to estimate gene differential expression (Supplemental Table 3).

\section{MicroRNA target predictions and enrichment analyses}

MicroRNA target sites in the annotated 3' UTR of T. castaneum protein-coding genes (r4.0, iBeetle Database, http://ibeetle-base. uni-goettingen.de/) were predicted using two independent target prediction algorithms with the default parameters-an implementation of the canonical site target pairing as described in Bartel (2009), provided by Antonio Marco, and miRanda (Enright et al. 2003). When multiple transcripts per gene were present $(7 \%$ of all annotations), different UTRs were merged; we note that considering individual transcripts separately produces very similar results (data not shown). Target enrichment was independently assessed by hypergeometric (phyper R function) and permutation tests. For the latter, random samples of equal sizes to the significantly up- and down-regulated gene sets between oocytes and later stages were drawn without replacement 1000 times, and numbers of microRNA targets were calculated. $P$-values were corrected for multiple testing using the Bonferroni correction. Sequence motif enrichment in transcript UTRs was assessed by sylamer (van Dongen et al. 2008).

\section{Data access}

All RNA sequencing data from this study have been submitted to the NCBI Gene Expression Omnibus (GEO; http://www.ncbi.nlm .nih.gov/geo/) under accession number GSE63770.

\section{Acknowledgments}

We thank Mario Stanke and Gregor Bucher for providing the T. castaneum $\mathrm{r} 4.0$ genome assembly and protein-coding gene annotations. This work was supported by the Wellcome Trust (093161/Z/10/Z) and the Biotechnology and Biological Sciences Research Council (BB/M011275/1).

Author contributions: M.N., M.R., and S.G.-J. conceived the study, designed the experiments, and wrote the manuscript. M.N. performed all experiments and data analyses and drafted the manuscript.

\section{References}

Altschul SF, Gish W, Miller W, Myers EW, Lipman DJ. 1990. Basic local alignment search tool. J Mol Biol 215: 403-410.

Anders S, Huber W. 2010. Differential expression analysis for sequence count data. Genome Biol 11: R106.

Anders S, Pyl PT, Huber W. 2015. HTSeq-a Python framework to work with high-throughput sequencing data. Bioinformatics 31: 166-169.

Asgari S. 2013. MicroRNA functions in insects. Insect Biochem Mol Biol 43: 388-397.

Bartel DP. 2004. MicroRNAs: genomics, biogenesis, mechanism, and function. Cell 116: 281-297.

Bartel DP. 2009. MicroRNAs: target recognition and regulatory functions. Cell 136: 215-233.

Baskerville S, Bartel DP. 2005. Microarray profiling of microRNAs reveals frequent coexpression with neighboring miRNAs and host genes. RNA 11: 241-247.

Benton MA, Akam M, Pavlopoulos A. 2013. Cell and tissue dynamics during Tribolium embryogenesis revealed by versatile fluorescence labeling approaches. Development 140: 3210-3220.

Biemar F, Zinzen R, Ronshaugen M, Sementchenko V, Manak JR, Levine MS 2005. Spatial regulation of microRNA gene expression in the Drosophila embryo. Proc Natl Acad Sci 102: 15907-15911.

Bissels UTE, Wild S, Tomiuk S, Holste A, Hafner M, Tuschl T, Bosio A. 2009. Absolute quantification of microRNAs by using a universal reference. RNA 15: 2375-2384.

Brennecke J, Malone CD, Aravin AA, Sachidanandam R, Stark A, Hannon GJ. 2008. An epigenetic role for maternally inherited piRNAs in transposon silencing. Science 322: 1387-1392.

Bushati N, Stark A, Brennecke J, Cohen SM. 2008. Temporal reciprocity of miRNAs and their targets during the maternal-to-zygotic transition in Drosophila. Curr Biol 18: 501-506.

Chen PY, Manninga H, Slanchev K, Chien M, Russo JJ, Ju J, Sheridan R, John B, Marks DS, Gaidatzis D, et al. 2005. The developmental miRNA profiles of zebrafish as determined by small RNA cloning. Genes Dev 19: 1288-1293. 
Denell R. 2004. Establishment of Tribolium as a genetic model system and its early contributions to evo-devo. Genetics 180: 1779-1786.

Domazet-Lošo T, Tautz D. 2010. A phylogenetically based transcriptome age index mirrors ontogenetic divergence patterns. Nature 468: $815-818$.

Enright AJ, John B, Gaul U, Tuschl T, Sander C, Marks DS. 2003. MicroRNA targets in Drosophila. Genome Biol 5: R1.

Friedländer MR, Chen W, Adamidi C, Maaskola J, Einspanier R, Knespel S, Rajewsky N. 2008. Discovering microRNAs from deep sequencing data using miRDeep. Nat Biotechnol 26: 407-415.

Giraldez AJ, Mishima Y, Rihel J, Grocock RJ, Van Dongen S, Inoue K, Enright AJ, Schier AF. 2006. Zebrafish MiR-430 promotes deadenylation and clearance of maternal mRNAs. Science 312: 75-79.

Griffiths-Jones S. 2005. RALEE-RNA ALignment editor in Emacs. Bioinformatics 21: 257-259.

Ha M, Kim VN. 2014. Regulation of microRNA biogenesis. Nat Rev Mol Cell Biol 15: 509-524.

Handel K, Grünfelder CG, Roth S, Sander K. 2000. Tribolium embryogenesis: a SEM study of cell shapes and movements from blastoderm to serosal closure. Dev Genes Evol 210: 167-179.

Handel K, Basal A, Fan X, Roth S. 2005. Tribolium castaneum twist: gastrulation and mesoderm formation in a short-germ beetle. Dev Genes Evol 215: $13-31$.

Heyn P, Kircher M, Dahl A, Kelso J, Tomancak P, Kalinka AT, Neugebauer KM. 2014. The earliest transcribed zygotic genes are short, newly evolved, and different across species. Cell Rep 6: 285-292.

$\mathrm{Hu}$ W, Criscione F, Liang S, Tu Z. 2014. MicroRNAs of two medically important mosquito species: Aedes aegypti and Anopheles stephensi. Insect Mo Biol 24: 240-252.

Kalinka AT, Varga KM, Gerrard DT, Preibisch S, Corcoran DL, Jarrells J, Ohler U, Bergman CM, Tomancak P. 2010. Gene expression divergence recapitulates the developmental hourglass model. Nature 468: 811-814.

Kim HS, Murphy T, Xia J, Caragea D, Park Y, Beeman RW, Lorenzen MD, Butcher S, Manak JR, Brown SJ. 2010. BeetleBase in 2010: revisions to provide comprehensive genomic information for Tribolium castaneum. Nucleic Acids Res 38: D437-D442.

Kloosterman WP, Plasterk RHA. 2006. The diverse functions of microRNAs in animal development and disease. Dev Cell 11: 441-450.

Kosman D, Mizutani CM, Lemons D, Cox WG, McGinnis W, Bier E. 2004. Multiplex detection of RNA expression in Drosophila embryos. Science 305: 846 .

Kozomara A, Griffiths-Jones S. 2014. miRBase: annotating high confidence microRNAs using deep sequencing data. Nucleic Acids Res 42: D68-D73.

Lai EC, Burks C, Posakony JW. 1998. The K box, a conserved 3' UTR sequence motif, negatively regulates accumulation of enhancer of split complex transcripts. Development 125: 4077-4088.

Lai EC, Tomancak P, Williams RW, Rubin GM. 2003. Computational identification of Drosophila microRNA genes. Genome Biol 4: R42.

Langmead B, Trapnell C, Pop M, Salzberg SL. 2009. Ultrafast and memoryefficient alignment of short DNA sequences to the human genome. Genome Biol 10: R25.

Lee RC, Feinbaum RL, Ambros V. 1993. The C. elegans heterochronic gene lin-4 encodes small RNAs with antisense complementarity to lin-14. Cell 75: 843-854

Lee M, Choi Y, Kim K, Jin H, Lim J, Nguyen TA, Yang J, Jeong M, Giraldez AJ, Yang H, et al. 2014. Adenylation of maternally inherited microRNAs by Wispy. Mol Cell 56: 696-707.

Leviten MW, Lai EC, Posakony JW. 1997. The Drosophila gene Bearded encodes a novel small protein and shares $3^{\prime}$ UTR sequence motifs with multiple Enhancer of split Complex genes. Development 124: 4039-4051.

Liang H, Li W-H. 2009. Lowly expressed human microRNA genes evolve rapidly. Mol Biol Evol 26: 1195-1198.

Lim LP, Glasner ME, Yekta S, Burge CB, Bartel DP. 2003a. Vertebrate microRNA genes. Science 299: 1540 .

Lim LP, Lau NC, Weinstein EG, Abdelhakim A, Yekta S, Rhoades MW, Burge CB, Bartel DP. 2003b. The microRNAs of Caenorhabditis elegans. Genes Dev 17: 991-1008.

Lowe TM, Eddy SR. 1997. tRNAscan-SE: a program for improved detection of transfer RNA genes in genomic sequence. Nucleic Acids Res 25: 955-964.

Lund E, Liu M, Hartley RS, Sheets MD, Dahlberg JE. 2009. Deadenylation of maternal mRNAs mediated by miR-427 in Xenopus laevis embryos. $R N A$ 15: $2351-2363$.

Marco A, Hui JHL, Ronshaugen M, Griffiths-Jones S. 2010. Functional shifts in insect microRNA evolution. Genome Biol Evol 2: 686-696.
Meunier J, Lemoine F, Soumillon M, Liechti A, Weier M, Guschanski K, Hu H, Khaitovich P, Kaessmann H. 2013. Birth and expression evolution of mammalian microRNA genes. Genome Res 23: 34-45.

Mito T, Nakamura T, Noji S. 2010. Evolution of insect development: to the hemimetabolous paradigm. Curr Opin Genet Dev 20: 355-361.

Nawrocki EP, Kolbe DL, Eddy SR. 2009. Infernal 1.0: inference of RNA alignments. Bioinformatics 25: 1335-1337.

Ninova M, Ronshaugen M, Griffiths-Jones S. 2014. Fast-evolving microRNAs are highly expressed in the early embryo of Drosophila virilis. RNA 20: $360-372$.

Ohnishi Y, Totoki Y, Toyoda A, Watanabe T, Yamamoto Y, Tokunaga K, Sakaki Y, Sasaki H, Hohjoh H. 2010. Small RNA class transition from siRNA/piRNA to miRNA during pre-implantation mouse development. Nucleic Acids Res 38: 5141-5151.

Pancratov R, Peng F, Smibert P, Yang S, Olson ER, Guha-Gilford C, Kapoor AJ, Liang F-X, Lai EC, Flaherty MS, et al. 2013. The miR-310/13 cluster antagonizes $\beta$-catenin function in the regulation of germ and somatic cell differentiation in the Drosophila testis. Development 140: 2904-2916.

Peel AD. 2008. The evolution of developmental gene networks: lessons from comparative studies on holometabolous insects. Philos Trans R Soc Lond B Biol Sci 363: 1539-1547.

Quint M, Drost H-G, Gabel A, Ullrich KK, Bönn M, Grosse I. 2012. A transcriptomic hourglass in plant embryogenesis. Nature 490: $98-101$.

Reinhart BJ, Slack FJ, Basson M, Pasquinelli AE, Bettinger JC, Rougvie AE Horvitz HR, Ruvkun G. 2000. The 21-nucleotide let-7 RNA regulates developmental timing in Caenorhabditis elegans. Nature 403: 901-906.

Richards S, Gibbs RA, Weinstock GM, Brown SJ, Denell RE, Beeman RW Bucher G, Friedrich M, Grimmelikhuijzen CJP, Klingler M, et al. 2008. The genome of the model beetle and pest Tribolium castaneum. Nature 452: 949-955.

Roth S, Hartenstein V. 2008. Development of Tribolium castaneum. Dev Genes Evol 218: 115-118.

Roux J, Gonzàlez-Porta M, Robinson-Rechavi M. 2012. Comparative analysis of human and mouse expression data illuminates tissue-specific evolutionary patterns of miRNAs. Nucleic Acids Res 40: 5890-5900.

Ruby JG, Stark A, Johnston WK, Kellis M, Bartel D, Lai EC. 2007. Evolution, biogenesis, expression, and target predictions of a substantially expanded set of Drosophila microRNAs. Genome Res 17: 1850-1864.

Ryazansky SS, Gvozdev VA, Berezikov E. 2011. Evidence for post-transcriptional regulation of clustered microRNAs in Drosophila. BMC Genomics 12: 371 .

Sarrazin AF, Peel AD, Averof M. 2012. A segmentation clock with two-segment periodicity in insects. Science 336: 338-341.

Schindelin J, Arganda-Carreras I, Frise E, Kaynig V, Longair M, Pietzsch T, Preibisch S, Rueden C, Saalfeld S, Schmid B, et al. 2012. Fiji: an opensource platform for biological-image analysis. Nat Methods 9: 676-682.

Tadros W, Lipshitz HD. 2009. The maternal-to-zygotic transition: a play in two acts. Development 136: 3033-3042.

Trapnell C, Pachter L, Salzberg SL. 2009. TopHat: discovering splice junctions with RNA-Seq. Bioinformatics 25: 1105-1111.

Trapnell C, Williams BA, Pertea G, Mortazavi A, Kwan G, van Baren MJ, Salzberg SL, Wold BJ, Pachter L. 2010. Transcript assembly and quantification by RNA-Seq reveals unannotated transcripts and isoform switching during cell differentiation. Nat Biotechol 28: 511-515.

Tsurudome K, Tsang K, Liao EH, Ball R, Penney J, Yang J-S, Elazzouzi F, He T, Chishti A, Lnenicka G, et al. 2010. The Drosophila miR-310 cluster negatively regulates synaptic strength at the neuromuscular junction. Neuron 68: 879-893.

van Dongen S, Abreu-Goodger C, Enright AJ. 2008. Detecting microRNA binding and siRNA off-target effects from expression data. Nat Methods 5: $1023-1025$.

Watanabe T, Takeda A, Mise K, Okuno T, Suzuki T, Minami N, Imai H. 2005. Stage-specific expression of microRNAs during Xenopus development. FEBS Lett 579: 318-324.

Zondag L, Dearden PK, Wilson MJ. 2012. Deep sequencing and expression of microRNAs from early honeybee (Apis mellifera) embryos reveals a role in regulating early embryonic patterning. BMC Evol Biol 12: 211.

Received April 21, 2015; accepted in revised form October 20, 2015.
96 Genome Research

www.genome.org 


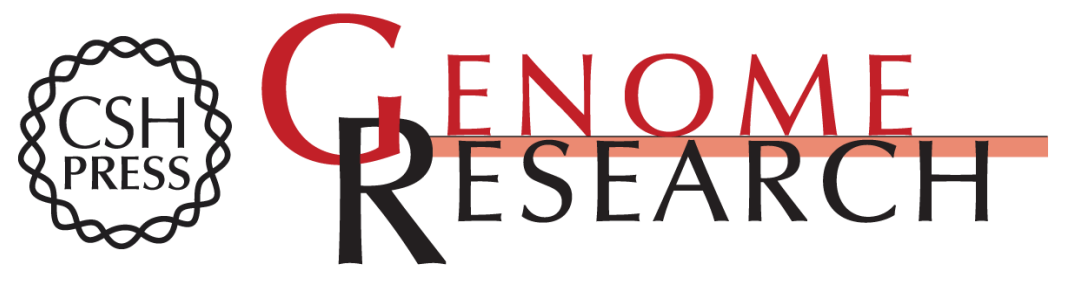

\section{MicroRNA evolution, expression, and function during short germband development in Tribolium castaneum}

Maria Ninova, Matthew Ronshaugen and Sam Griffiths-Jones

Genome Res. 2016 26: 85-96 originally published online October 30, 2015

Access the most recent version at doi:10.1101/gr.193367.115

Supplemental Material

References

Open Access

Creative Commons

License

Email Alerting Service
http://genome.cshlp.org/content/suppl/2015/10/27/gr.193367.115.DC1

This article cites 64 articles, 19 of which can be accessed free at: http://genome.cshlp.org/content/26/1/85.full.html\#ref-list-1

Freely available online through the Genome Research Open Access option.

This article, published in Genome Research, is available under a Creative Commons License (Attribution 4.0 International), as described at http://creativecommons.org/licenses/by/4.0/.

Receive free email alerts when new articles cite this article - sign up in the box at the top right corner of the article or click here.

\section{Affordable, Accurate Sequencing.}

